\title{
Impact of MODIS SWIR band calibration improvements on Level-3 atmospheric products
}

\author{
Andrew Wald ${ }^{1}$, Robert Levy ${ }^{2}$ Amit Angal ${ }^{3}$, Xu Geng $^{3}$, Jack Xiong ${ }^{2}$, Kurt Hoffman ${ }^{3}$ \\ ${ }^{1}$ Global Science and Technology, Greenbelt, Maryland 20770 \\ ${ }^{2}$ NASA Goddard Space Flight Center, Greenbelt, MD, 20771 USA \\ ${ }^{3}$ Science Systems and Applications Inc. Lanham, Maryland 20706
}

\begin{abstract}
The spectral reflectance measured by the MODIS reflective solar bands (RSB) is used for retrieving many atmospheric science products. The accuracy of these products depends on the accuracy of the calibration of the RSB. To this end, the RSB of the MODIS instruments are primarily calibrated on-orbit using regular solar diffuser (SD) observations. For $\lambda$ $<0.94 \mu \mathrm{m}$ the SD's on-orbit bi-directional reflectance factor (BRF) change is tracked using solar diffuser stability monitor (SDSM) observations. For $\lambda>0.94 \mu \mathrm{m}$, the MODIS Characterization Support Team (MCST) developed, in MODIS Collection 6 (C6), a time-dependent correction using observations from pseudo-invariant earth-scene targets. This correction has been implemented in C6 for the Terra MODIS $1.24 \mu \mathrm{m}$ band over the entire mission, and for the $1.375 \mu \mathrm{m}$ band in the forward processing. As the instruments continue to operate beyond their design lifetime of six years, a similar correction is planned for other short-wave infrared (SWIR) bands as well.

MODIS SWIR bands are used in deriving atmosphere products, including aerosol optical thickness, atmospheric total column water vapor, cloud fraction and cloud optical depth. The SD degradation correction in Terra bands 5 and 26 impact the spectral radiance and therefore the retrieval of these atmosphere products. Here, we describe the corrections to Bands $5(1.24 \mu \mathrm{m})$ and $26(1.375 \mu \mathrm{m})$, and produce three sets (B5, B26 correction $=$ on/on, on/off, and off/off) of Terra-MODIS Level 1B (calibrated radiance product) data. By comparing products derived from these corrected and uncorrected Terra MODIS Level 1B (L1B) calibrations, dozens of L3 atmosphere products are surveyed for changes caused by the corrections, and representative results are presented. Aerosol and water vapor products show only small local changes, while some cloud products can change locally by $>10 \%$, which is a large change.
\end{abstract}

Keywords: remote sensing, MODIS, calibration, aerosol, water vapor, cloudmask, cirrus.

\section{INTRODUCTION}

MODIS spectral reflectance is used to derive many atmospheric science products, but the accuracy of these products depends on accurate calibration. The MODIS reflective solar bands (RSB) are calibrated on-orbit using regular solar diffuser (SD) observations, with the SD's on-orbit bi-directional reflectance factor change tracked using solar diffuser stability monitor (SDSM) observations ${ }^{1,2}$. The instrument gain for each band, detector, subframe and mirror side is characterized by observing the [known-reflectance] SD via

$$
\rho_{\mathrm{SD}} \cos \left(\theta_{\mathrm{SD}}\right)=\mathrm{m} 1_{\mathrm{SD}} \mathrm{dn}_{\mathrm{SDES}}{ }^{2}(1+\mathrm{kT}) / \mathrm{RVS}
$$

where $\rho_{\mathrm{SD}}$ is the target reflectance, $\cos \left(\theta_{\mathrm{SD}}\right)$ is the solar zenith angle, $\mathrm{m} 1$ is the inverse gain of the instrument, $\mathrm{dn}$ is the digital number, and $\mathrm{D}_{\mathrm{ES}}$ is the Earth-sun distance in $\mathrm{AU},(1+\mathrm{kT})$ is an instrument temperature-dependent factor, and RVS is the Response Vs Scan angle, to account for changing scan mirror reflectance as a function of scan angle ${ }^{3}$. The scan mirror is double-sided with separate calibration for each side. Bands 5-7 have 20 detectors each with 500-m ground resolution, and $\mathrm{B} 26$ has 10 detectors with $1 \mathrm{~km}$ resolution. In the case of Aqua MODIS band 6, several detectors were identified to be inoperable during the prelaunch testing. Also, the signal-to-noise ratio (SNR) for the Terra band 7 detectors 
does not meet the specification ${ }^{4}$. The SWIR bands in both MODIS instruments have a known impact due to electronic crosstalk and optical leak. This behavior was identified prior to launch and has more impact on the Terra MODIS SWIR bands than on Aqua MODIS. A correction based on night-time observations of earth-scenes has been implemented since launch for both instruments. The change in estimated SD reflectance due to this additional correction for its degradation changes estimated instrument gain. This change in gain then changes all derived top-of-atmosphere B5 and B26 reflectances and the science products that depend on them.

The SDSM can only monitor the SD reflectance at wavelengths $\lambda<0.94 \mu \mathrm{m}$. Figure 1 shows the SDSM-measured spectral dependence of SD degradation for three different years during the mission ${ }^{5}$.

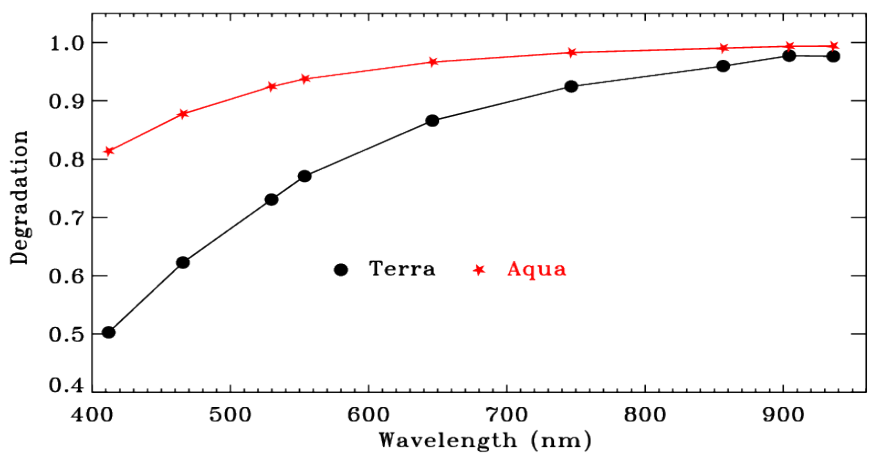

Figure 1. SD reflectance degradation at $\lambda<0.94 \mu \mathrm{m}$ for 2014 for Terra (black) and Aqua (red). Exposure of the SD to the harsh space environment, including solar UV, significantly degrades its reflectance. The Terra SD degradation is larger because it has been on orbit two years longer, and because its SD door has been in the open position since 2003, while the Aqua SD door is open only once every three weeks. In the visible this degradation can reach $50 \%$. At $\lambda>0.94$, not measured by the SDSM, the degradation as estimated by observations of pseudo-invariant earth sites is approximately $1 \%$.

With Terra MODIS entering its $16^{\text {th }}$ year on orbit the SD degradation at longer wavelengths is no longer negligible. The SDSM degradation at $\lambda>0.94 \mu \mathrm{m}$ is now monitored via observations of pseudo-invariant earth view sites such as desert and deep convective clouds [section 2 below], with long-term trending from these sites used to estimate the degradation at these wavelengths. Because the Terra MODIS SD door has been open since 2003, its SD degradation has been much larger than the Aqua MODIS SD degradation, whose door is open only once every three weeks for calibration.

\section{REFLECTANCE CORRECTION}

Figure 2 shows C6 band-averaged reflectance trending results (without accounting the SD degradation at this wavelength) from the Libya 4 site for Band 5 for mirror side one at frame 1040, which is the frame with available results nearest to frame 978, which is the SD frame [4]. The uptrend over mission is due to SD degradation and exceeds $1 \%$ over mission lifetime. It is observed to be the same for both mirror sides 


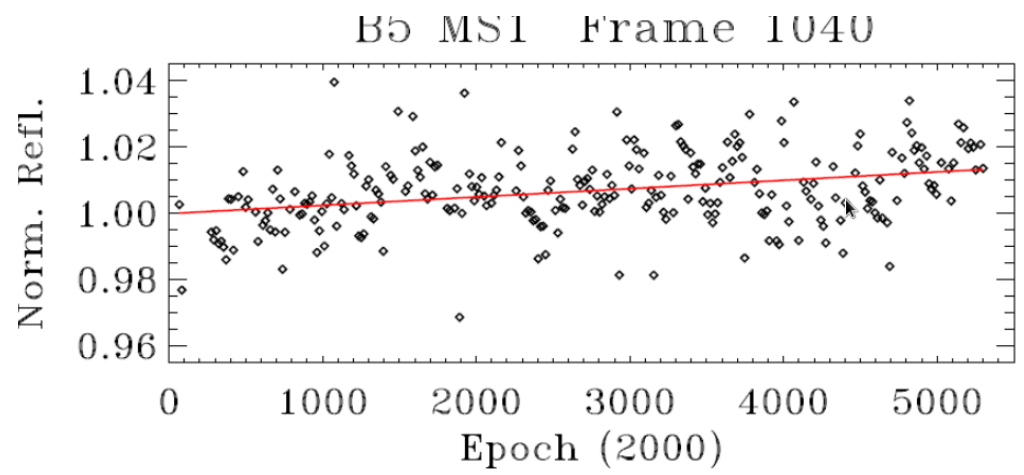

Figure 2. Reflectance rending from pseudo-invariant site. The uptrend is due to SD reflectance degradation.

The degradation can be expected to be exponential in nature with possible seasonal variation. Due to the small size of the correction, it is assumed in C6 to be well-approximated as linear in time. Based on observed desert trending, Terra Band 5 is corrected for SD degradation for a given day of mission [DOM] is given by

$$
\mathrm{r}_{\text {corr }}=\mathrm{r}_{\text {uncor }} *\left(1.0+\mathrm{DOM}^{*} 2.1875881 \times 10^{-6}\right)
$$

For Jan 1, $2016=$ DOM 5844, the correction factor is $1.2 \%$.

B26, the $1.38 \mu \mathrm{m}$ cirrus channel, does not see to ground due to strong water vapor absorption. But trending results based on deep convective clouds [6] can be used to find the trending. The B26 SD degradation correction as a linear function of DOM is

$$
\mathrm{r}_{\text {corr }}=\mathrm{r}_{\text {uncor }} *\left(1.0+\mathrm{DOM} * 7.0000 \times 10^{-7}\right)
$$

For Jan 1, 2016 the correction factor is $0.4 \%$.

Downstream atmospheric science products [cloudmask, aerosol, water vapor, clouds] are affected by the changed B5 and B26 reflectances, but these effects were not studied before the B5 and B26 corrections were implemented. The purpose of this work is to describe how atmospheric science products are affected by these corrections. Table 1 lists the categories of surveyed science products. Intermediate products and various statistical subcategories are not listed.

Table 1. Partial list of surveyed MOD08 L3 products.

\begin{tabular}{|l|l|l|}
\hline Aerosol & Water Vapor & Clouds \\
\hline Dark Target optical depth & $\begin{array}{l}\text { Total column water vapor from near - } \\
\text { IR }\end{array}$ & Cloud water path liquid \\
\hline Deep Blue optical depth & Total column water vapor from IR & Cloud water path ice \\
\hline Aerosol over water optical depth & & Cloud effective radius liquid \\
\hline Deep Blue Angstrom parameter & & Cloud effective radius \\
\hline & & Cloud optical thickness \\
\hline & & Cloud optical thickness ice \\
\hline & & Cloud emissivity \\
\hline & & Cloud fraction \\
\hline & & Cloud top temperature day \\
\hline
\end{tabular}




\section{APPLICATIONS TO MODIS DATA}

MODIS science products rely on calibrated reflectance data. In Collection 5 (C5) of the MODIS product, L1B calibration factors were calculated using measurements of the SD and the SDSM. Starting with Collection 6 (C6), the MODIS calibration relies on the Earth View (EV) targets to supplement the constraints provided by the SD and the SDSM, particularly at short wavelengths [4]. C6 processing began in 2011, so that degradation in longer wavelengths had not yet been detected. Currently the B5 correction is applied to the full mission and the B26 correction to the forward processing. As a MODIS collection should be a consistent application of a particular algorithm, including calibration, this means there is a possible discontinuity in MODIS data starting in 2015.

Two global sets of L1B granules were selected: (1) Jan, April, and July in 2005 ["early mission"] and (2) the same months in 2014 ["late mission"]. These months/years were chosen to observe possible seasonal variations in results, and to assess the impact on decadal trends. Table 2 lists the combinations of corrections applied.

Table 2. Table of corrections applied to L1B granules. The granules are identical except for these differences.

\begin{tabular}{|l|l|}
\hline Correction Applied & Used for \\
\hline B5 on, B26 off & C6 operational correction for the years tested \\
\hline B5 on, B26 on & C6 operational correction for $t \geq 2015$ \\
\hline B5 off, B26 off & for baseline testing \\
\hline
\end{tabular}

All versions of the granules are sent to the test facility at the MODIS Adaptive Processing System (MODAPS), which ran identical C6 processing streams (aerosol, cloud, and water vapor retrieval algorithms) on each version. The retrieved aerosol and cloud products (known as Level 2, or L2) are further processed into gridded statistics at $1^{\circ} \times 1^{\circ}$ resolution (known as Level 3, or L3). [7]. L3 are derived on daily (L3-D) and on monthly (L3-M) scales. Since all other inputs are identical, differences in the resulting science products must arise only from differences in applying the B5 and B26 corrections.

\section{Science products}

Bands 5 and 26 are inputs to many atmospheric science products. We surveyed more than one hundred intermediate and official atmospheric science products related to aerosol, water vapor, and cloud properties. Section 4 shows some representative results of some official MODIS science products. Some science products do not use B5 and B26 directly. But because B5 and B26 are used in the cloud mask, those science products may still be affected by the corrections.

\section{Aerosols}

There are three MODIS aerosol retrieval algorithms. They are the "dark-target" (DT) algorithm that is composed of subalgorithms over land (DT-L) and ocean (DT-O), and the Deep-Blue (DB) which also retrieves over land. These retrieval algorithms operate on clear-sky pixels (non-cloudy). The goal of these retrievals is to derive the contribution of the aerosol as aerosol optical depth (AOD). A necessary step to do this is to separate the aerosol radiance from the surface radiance. The nominal retrieval is AOD in the green $(\mathrm{B} 4 ; \lambda=0.55 \mu \mathrm{m})$, but this AOD is retrieved using data from other wavelengths. For example, DT-L uses reflectance in B1 $(\lambda=0.65 \mu \mathrm{m}), \mathrm{B} 3(0.47 \mu \mathrm{m})$ and B7 $(2.11 \mu \mathrm{m})$ to derive AOD in B4. The surface reflectance contribution is assumed to have a prescribed relationship based on the three bands, and the AOD is derived from the residual. However, the ratio of observed reflectance in B5 and B7 is used to fine-tune the assumed B1/B3/B7 surface relationship. B5 (along with B2; $\lambda=0.86 \mu \mathrm{m}$ ) is used to identify and mask surfaces with patchy snow cover (that are unsuitable for aerosol retrieval). Therefore, changes in B5 will affect the DT-L retrieval.

B5 is used for DT-O, in that it is one of six wavelengths (B1, B2, B4, B5, B6, and B7) used in an inversion to retrieve AOD. B5 is also used with B6 to filter out pixels along coastlines that may have underwater sediments. B5 is not used directly in DB retrieval, which is based on observations and assumptions in B1, B4, and B9 $(\lambda=0.41 \mu \mathrm{m})$.

B26, while not used directly, affects all aerosol retrieval algorithms. B26 is used to identify and/or filter out cirrus clouds that would otherwise contaminate the aerosol retrieval. For example, the dark-target retrieval over ocean uses B26 in two 
ways. It considers all pixels with B26 reflectance $\mathrm{r}(1.38)>0.03$ to be cloudy. At the same time, for cases where $\mathrm{r}(1.38)<0.03$, there are additional tests that can be applied (using other channels, including B5) to indicate that the scene might be cloudy and to assign flags for lowered confidence. Similarly, the other retrieval algorithms apply similar tests for B26, which means that correcting B26 can alter which pixels are classified as cirrus, and may result as changes in L3 aerosol statistics ${ }^{8,9,10}$.

\section{Water Vapor}

The water vapor algorithm uses B5 $1.24 \mu \mathrm{m}$ reflectance, along with reflectance at $0.86 \mu \mathrm{m}$ for interpolation to estimate true surface reflectance in the three MODIS water channels between them in the $0.94 \mu \mathrm{m}$ water vapor absorption feature. Therefore changes in estimated B5 reflectance propagate directly to changes in estimated total column water vapor ${ }^{11}$.

Also, the water vapor retrieval includes an aerosol correction module to account for absorption enhanced by multiple scattering. Therefore changes in aerosol, as described above, can propagate to total column water vapor [TCWV]. TCWV is retrieved over land and over sunglint ocean. The MODIS water vapor ATBD states "Of particular interest is the collection of water vapor data above cirrus clouds, which has important application to climate studies". TCWV is retrieved separately over surface and over clouds. Changes in cloud pixel classification therefore change TCWV L3 statistics.

\section{Clouds and Cloudmask}

B26 is the basis for one of two tests for cirrus detection. Changing the L1B B26 reflectance will directly change cirrus reflectance and therefore any science products that use cirrus reflectance thresholds.

Band 5 is used in several auxiliary tests in the MODIS MOD35 cloud mask. The MOD035 ATBD states "Smoke and pollution tests are performed over solar illuminated water surfaces. Smoke is indicated when the standard deviation of R0.86 over the pixel of interest and the surrounding eight pixels is $<0.003, \mathrm{R} 1.24$ is between 0.022 and $0.050, \mathrm{R} 0.47 \geq$ $0.12, \mathrm{R} 0.47 / \mathrm{R} 1.24 \geq 5.0$, and R2.1 / R1.24 < 0.5. In addition, smoke is indicated when the standard deviation of R0.86 $<0.003$, the simple R0.86 water threshold test indicated cloud, and R0.47 / R1.24 $\geq 2.5$. In cases where the above standard deviation criterion is not met, smoke may still be indicated if R0.47 / R1.24 $\geq 2.5$ and R2.1 / R.24 <0.3." ${ }^{12}$.

\section{RESULTS}

The MODAPS Level 3 science products for [B5on, B26on] are taken as the baseline results. [B5on, B26off] and [B5off, B26off] results are compared to baseline. Because the 2014 results more clearly show the correction effects, all results are shown for 2014 data. Daily results are for July 29, 2014 and monthly results are from Jan 2014. All baseline images are of [B5on, B26 on] and all difference images are [B5off, B26off] - [B5on, B26on]. In some cases where the difference might result from either band 5 or band 26, we also include [B5on, B26off] - [B5on, B26on].

\section{Aerosol}

Aerosol optical depth over land for one day is shown in Figure 3. Even though B5 is explicitly used in this retrieval, the change in L3 retrieved optical depth for this month is close to 0.0 . 

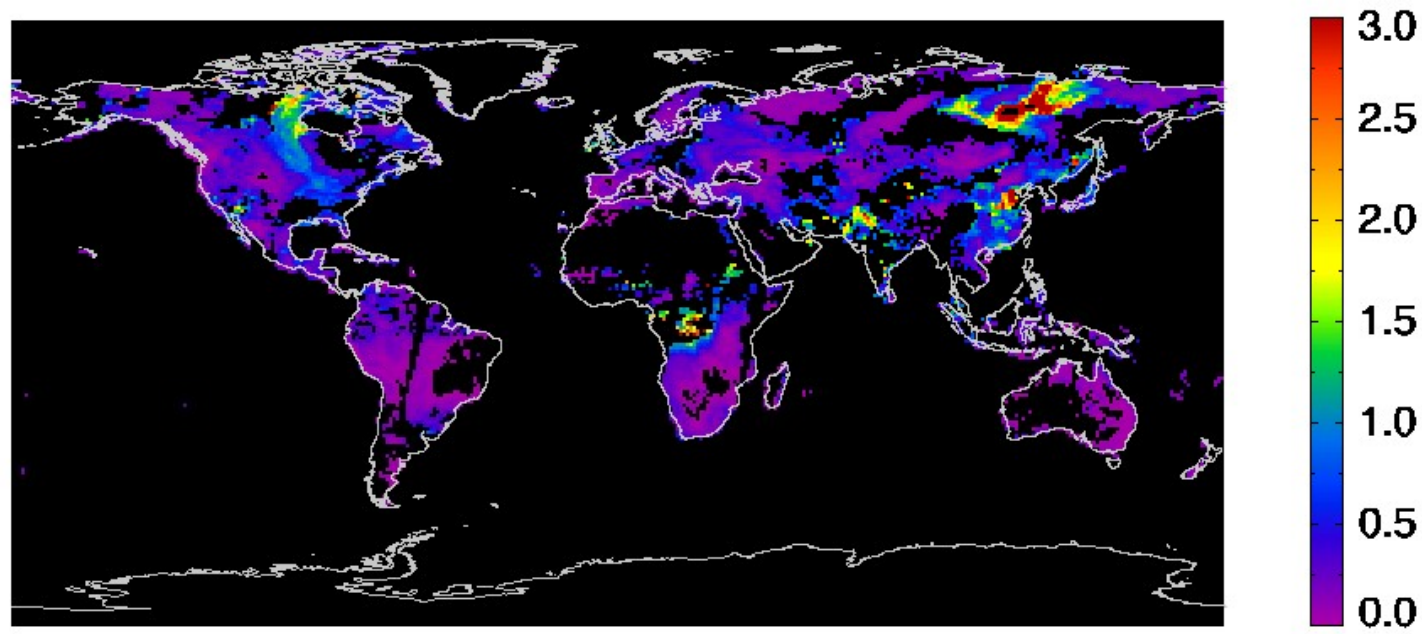

MOD08_D3.A2014212.006.2016037175341.hdf

units $=$ "none"

\section{Aerosol_Optical_Depth_Land_Mean}

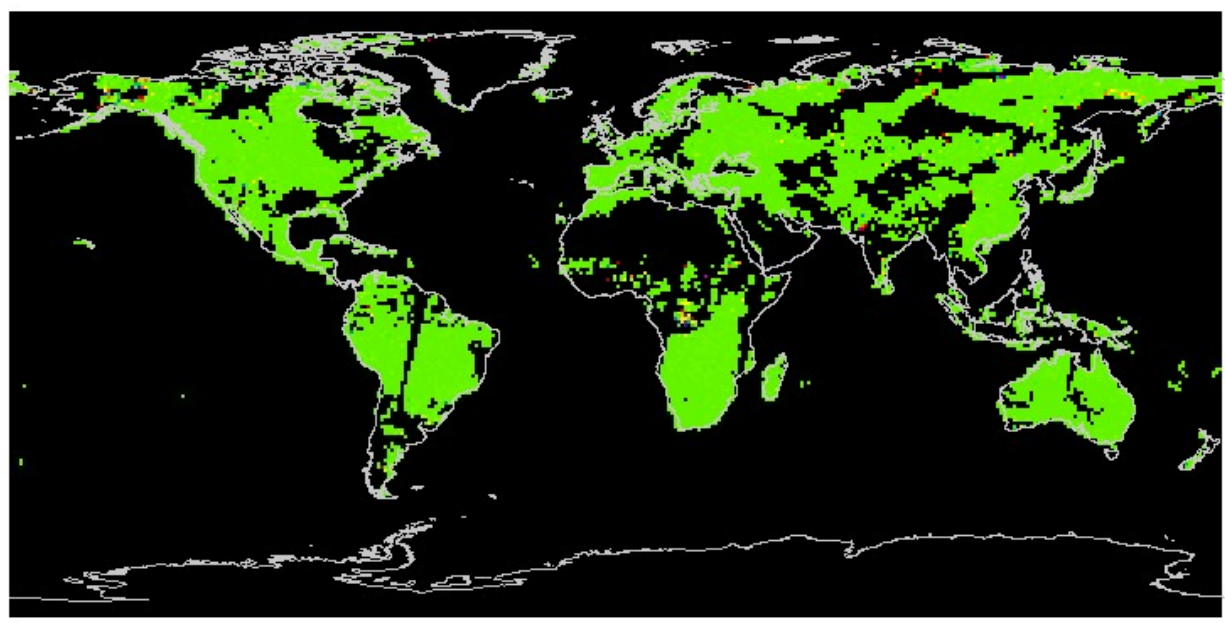

Test Data - Baseline Data
AS $1372-1371$

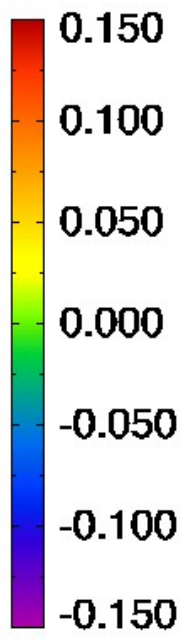

units = "none"

Figure 3. (a) Aerosol optical depth for July 29, 2014 with [B5off, B26off], and (b) difference in AOT due to [B5on, B26on]. The differences are close to zero almost everywhere. Archive Set 1372 refers to [B5off, B26off], 1371 to [B5on, B26on], and AS 6 to Collection 6 pre-2015 [B5on, B26off]. 
A closer look at the difference image shows some larger-value differences. These are clustered near, but not exclusively at, edges of coverage gaps [fig 4].

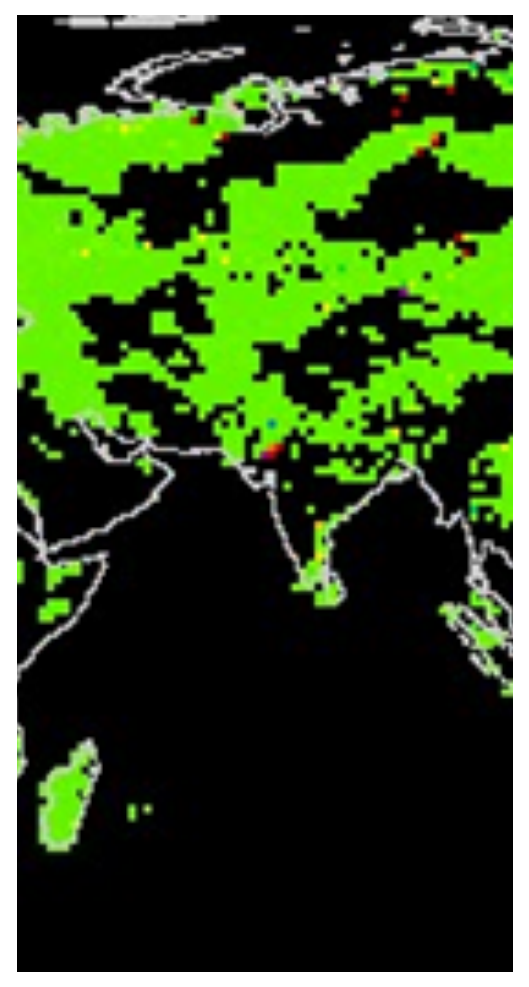

Figure 4. Aerosol optical depth change detail. The relatively large change [orange pixels in India] is likely due to changed number of pixels included in the retrieval, with resulting change in AOT.

Based on Figure 5, changes in the number of pixels used in retrievals is a key factor in understanding the B5 and B26 correction effect on aerosol.. The Dark Target aerosol algorithm uses 500-m and 1-km pixels in its retrieval. Therefore up to $20 \times 20=400 \mathrm{~L} 1 \mathrm{~B}$ pixels may be used in a given L2 retrieval, which operate on 10x10km grid cells. Pixels are rejected from retrieval based on cloudiness and underlying surface type and brightness. The pixel number plotted here is the mean of all the L2 retrievals used in the L3 grid cell. The B26 correction can change which pixels are classified as cloud, and therefore change the Aerosol_Number_Pixels_Used_Land_Mean and therefore change optical depth statistics, 

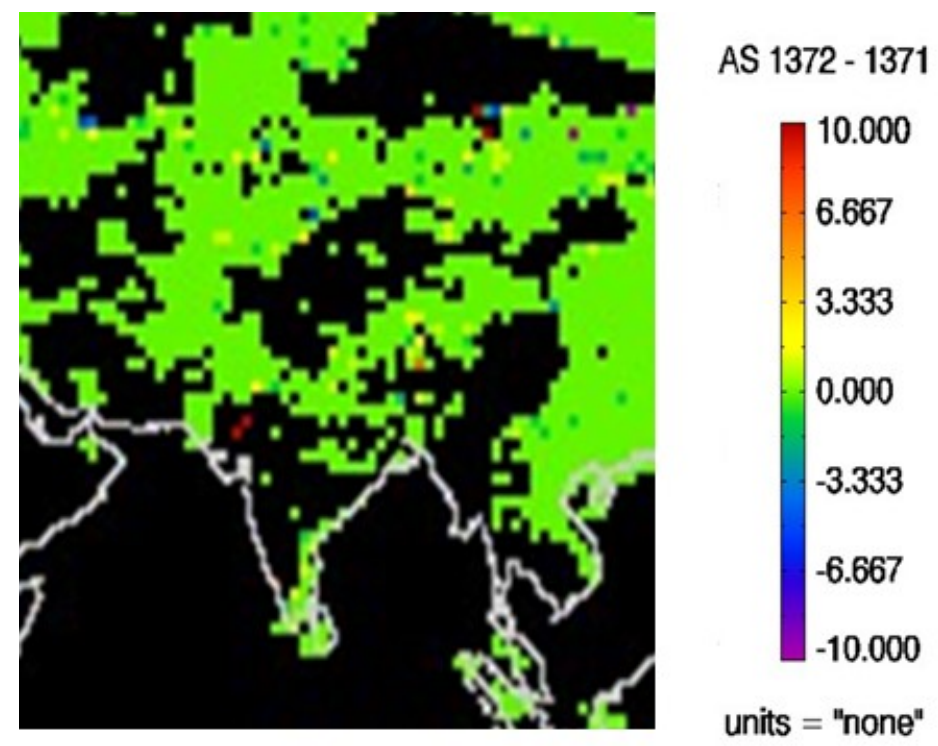

Figure 5 is the change in the number of pixels retrieved. The change in AOT in Figure 5 is related to the change in pixel number use din retrieval shown here.

Monthly aerosol results are shown in Figure 6. Globally, the correction effect for this month is very close to zero. Locally, off the east and west coast of Africa, results from the aerosol over water optical depth algorithm can change $\sim 0.01$. The similarity between the two difference maps means that the aerosol difference arises from changes in B26 reflectance, not B5.

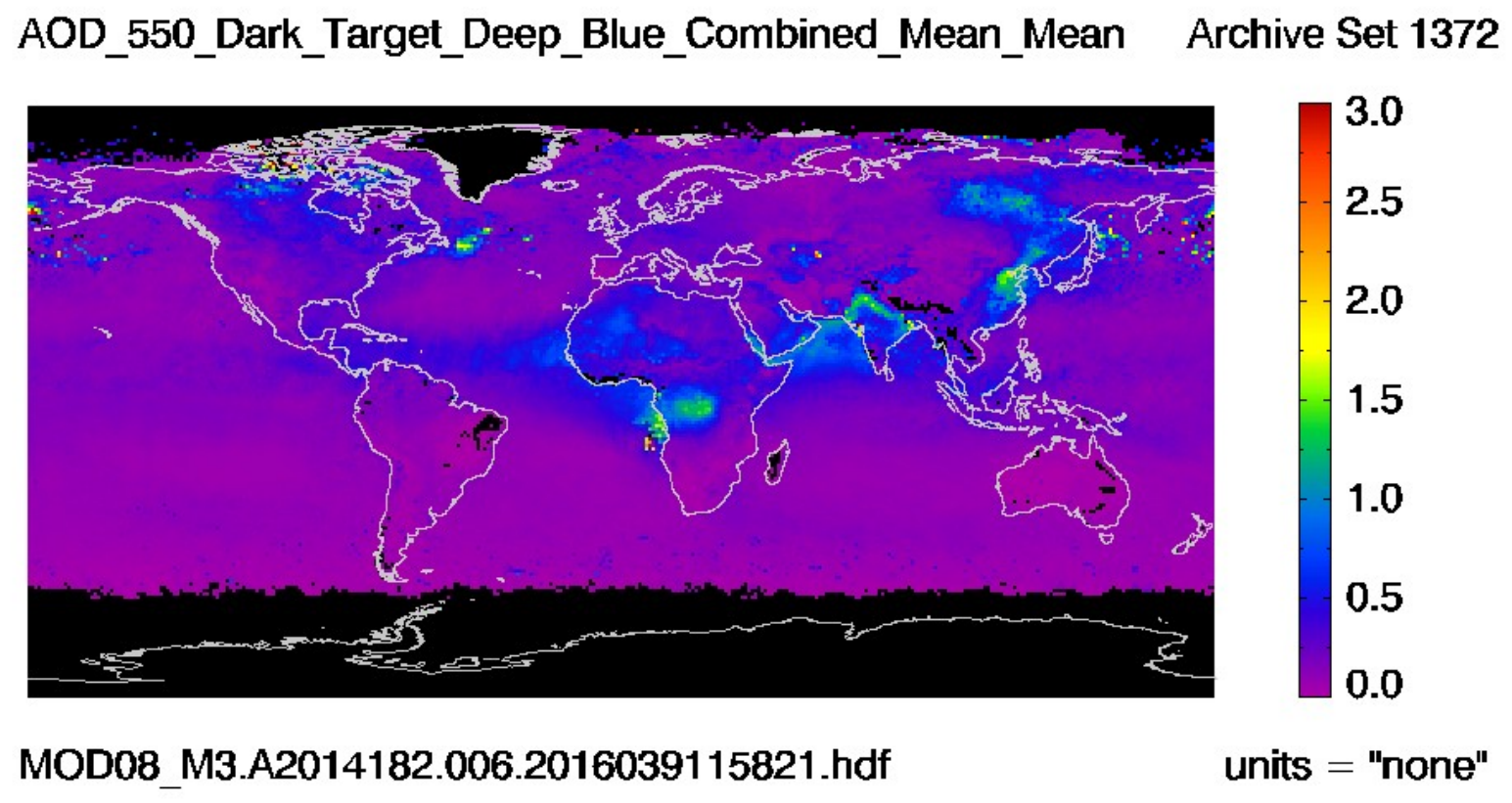




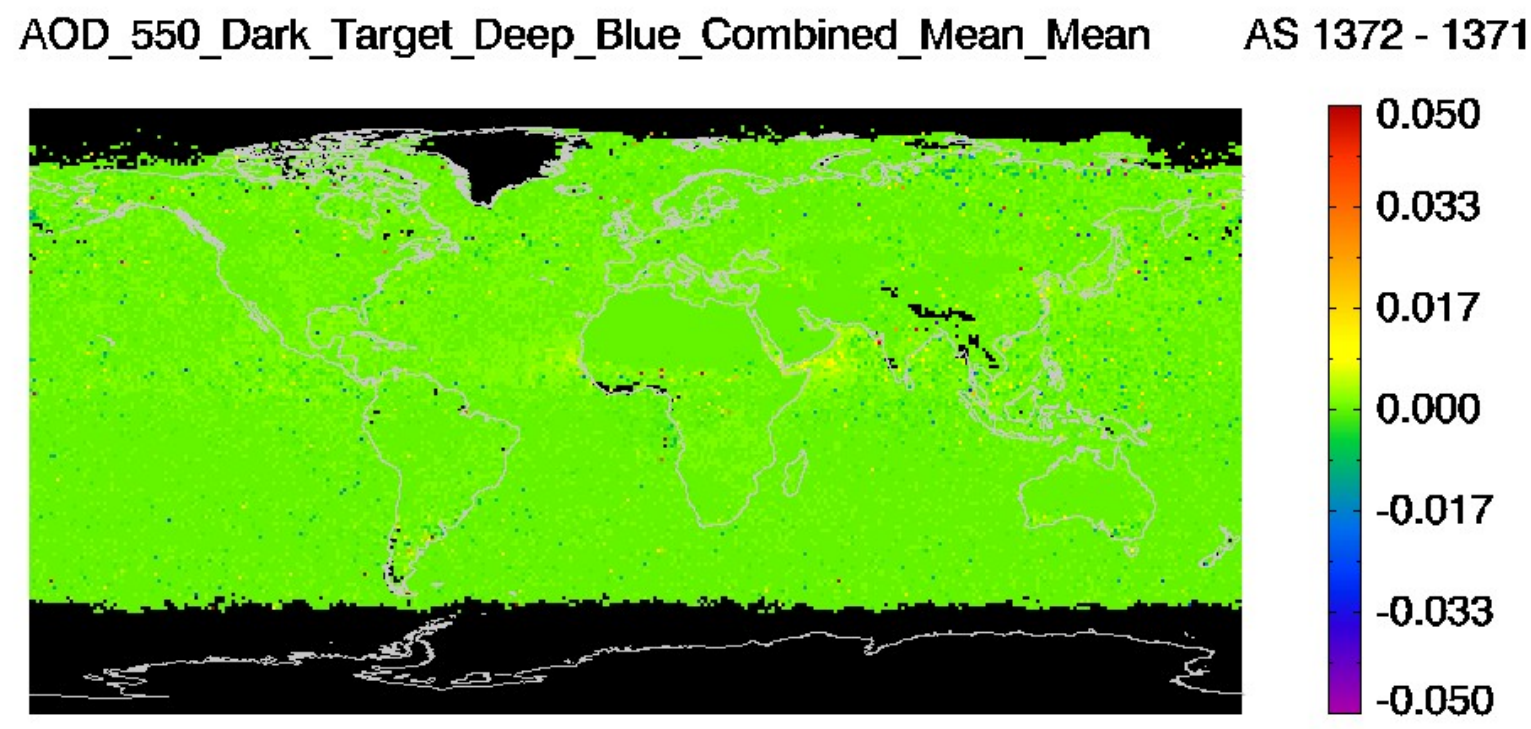

\section{Test Data - Baseline Data}
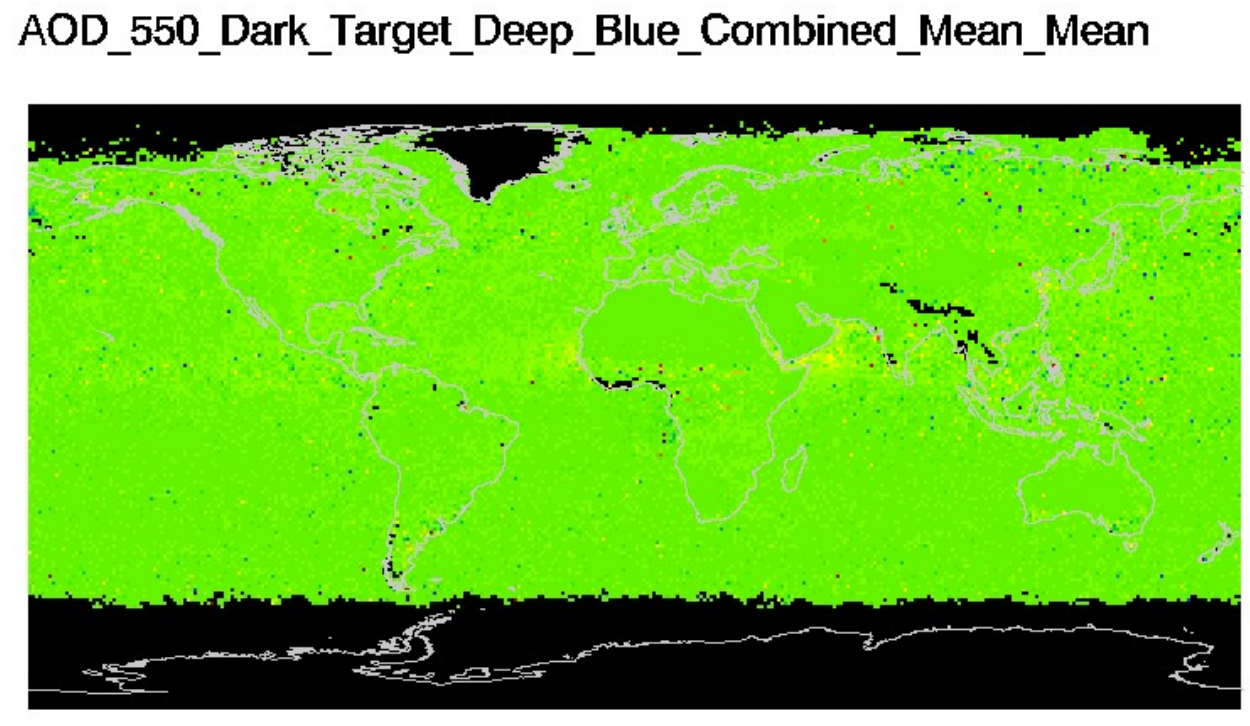

\section{Test Data - Baseline Data}

$$
\text { units = "none" }
$$

AS 1372 - 6

0.050

0.033

0.017

0.000

$-0.017$

$-0.033$

$-0.050$

units = "none"

Figure 6. (a) Monthly mean L3 aerosol with [B5off, B26off], and (b) difference image [B5off, B26off] - B5on, B26on], and (c) [B5off, B26off- B5on, B26off]. The main difference, which arises from B26, is off east and west coasts of Africa.

\section{Total column water vapor}

The Water_Vapor_Near_Infrared_Clear_Mean product uses band 5 at $1.24 \mu \mathrm{m}$ to help estimate surface reflectance in bands 17-19 near the $0.94 \mu \mathrm{m}$ water vapor absorption feature [5]. There is almost no change in derived TCWV due to the 
B5 and B26 changes [Fig 7]. The correction effect is uniform over land, and uniform over water, and slightly larger over water than land.

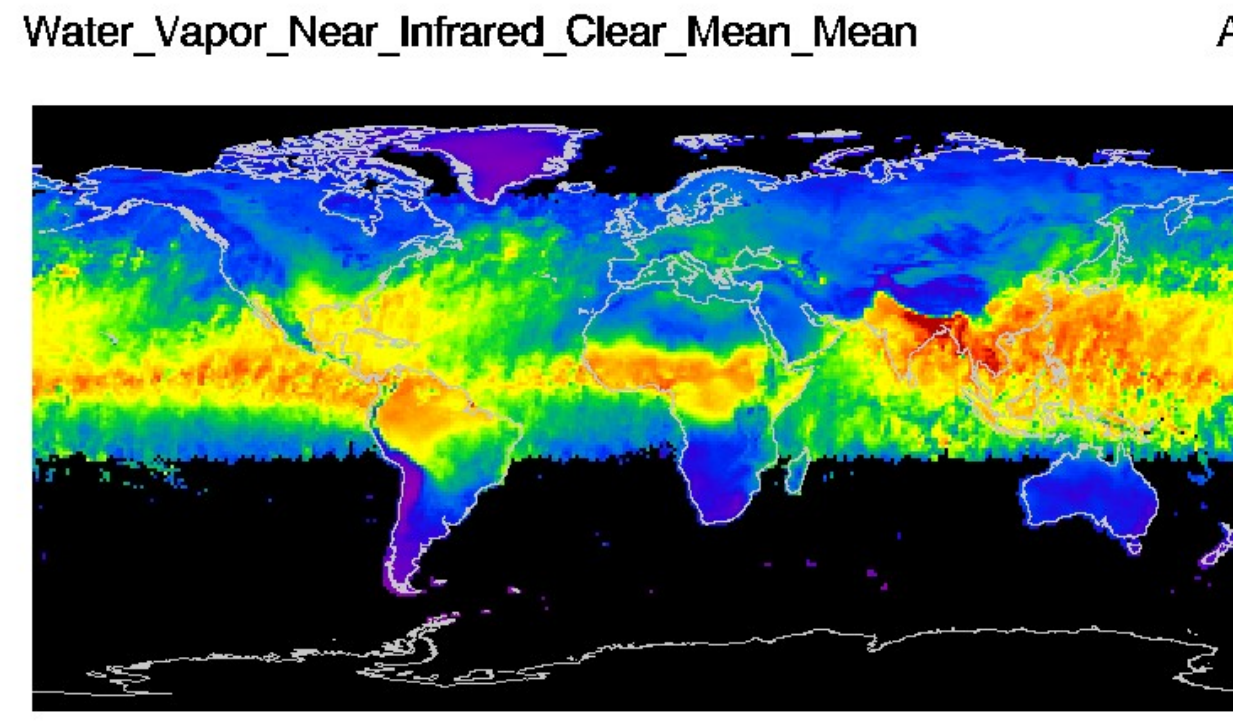

MOD08_M3.A2014182.006.2016039115821.hdf

Water_Vapor_Near_Infrared_Clear_Mean_Mean

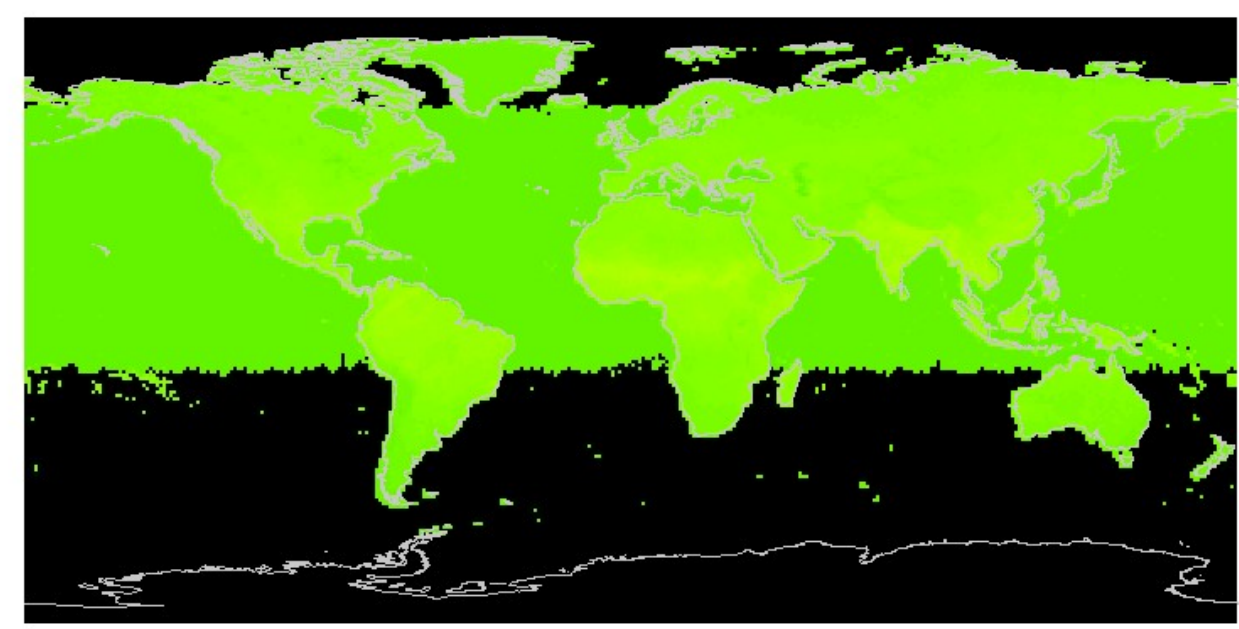

Test Data - Baseline Data
Archive Set 1372

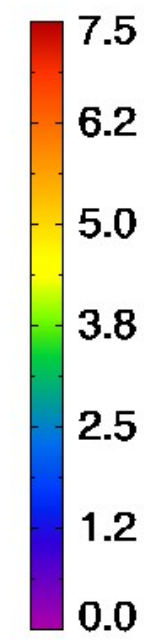

units $=$ "cm"

AS $1372-1371$

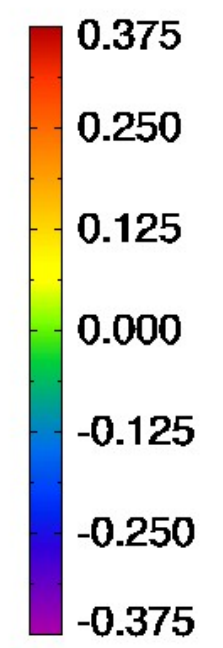

units $=$ "cm"

Figure 7 (a) Baseline monthly TCWV from NIR channels with [B5off, B26off] and (b) difference [B5off, B26off] - B5on, B26on].

Clouds 
Cirrus fraction, a threshold-based test, will be directly affected by changed B26. Figure 8 shows the monthly average cirrus fraction for Jan 2014, the [B5on, B26off] - [B5on B26 on] difference, and [B5off, B26off] - [B5on B26 on]. The effect of the B5 correction is opposite to the B26 correction. In both correction images, the cirrus fraction can be seen to increase in some places and decrease in others, e.g. over the Sahara and the Amazon.

Cirrus_Fraction_SWIR_FMean

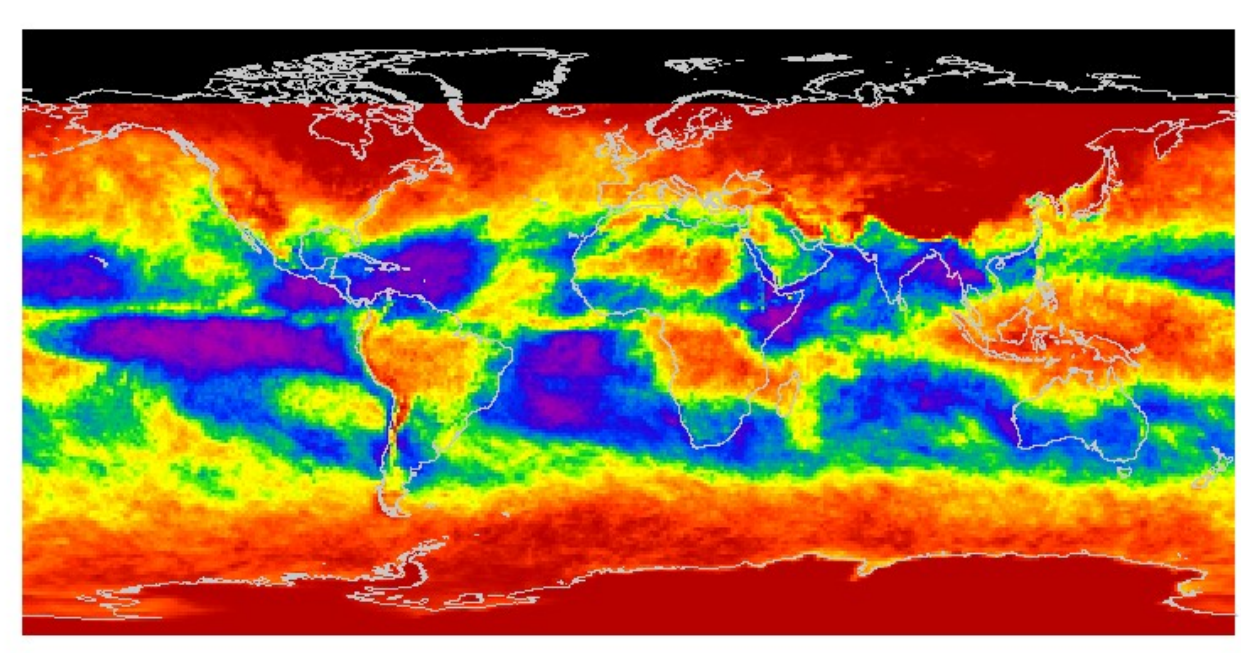

MOD08_M3.A2014001.006.2016037064008.hdf

Cirrus_Fraction_SWIR_FMean

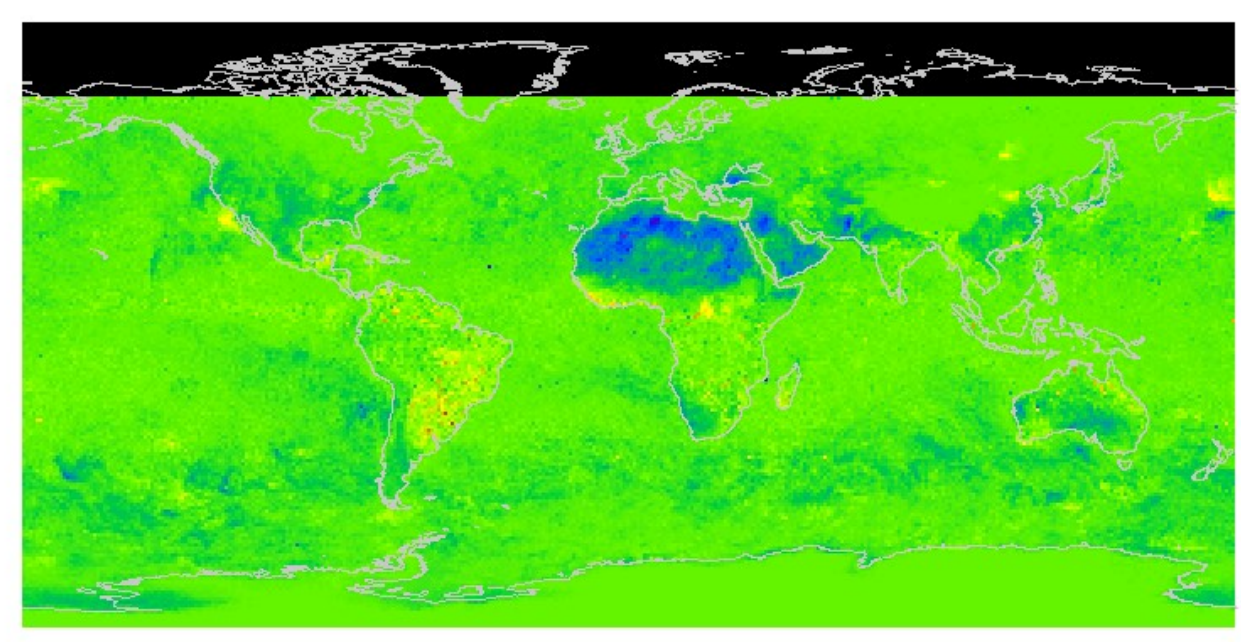

Test Data - Baseline Data
Archive Set 1372

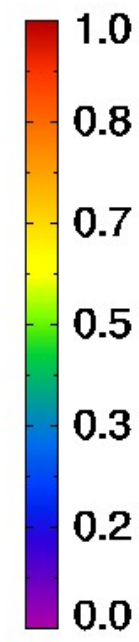

units $=$ "none"
AS 1372 - 6

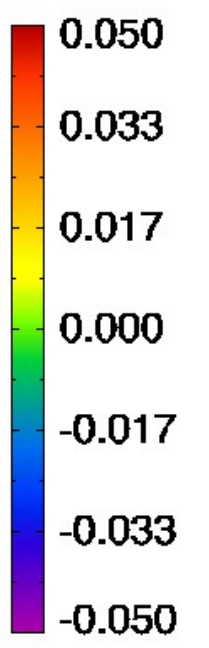

units = "none" 

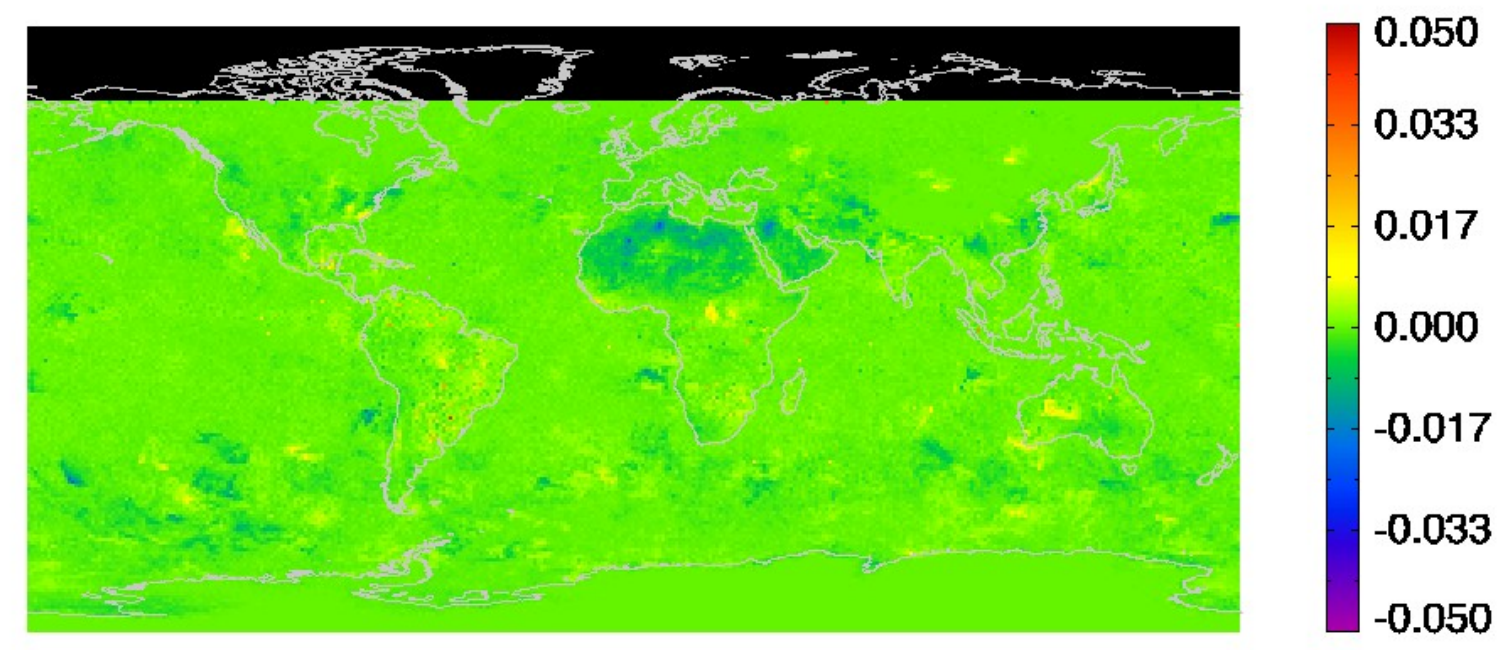

\section{Test Data - Baseline Data}

units = "none"

Figure 8. Cirrus fraction (a) (B5off, B26 off), (b) (B5off, B26 off) - (B5on, B26off). The difference is due to B5. (c) (B5off, B26off - B5on B26on). The differences in (c) are due to both B5 and B26. The difference between (b) and (c) is due to B26 correction effect. The B5 and B26 effects oppose each other.

Figure 9 shows the cloud liquid water path monthly mean. There can be a 10\% change in liquid water path near the poles.

\section{Cloud_Water_Path_16_Liquid_Mean_Mean}

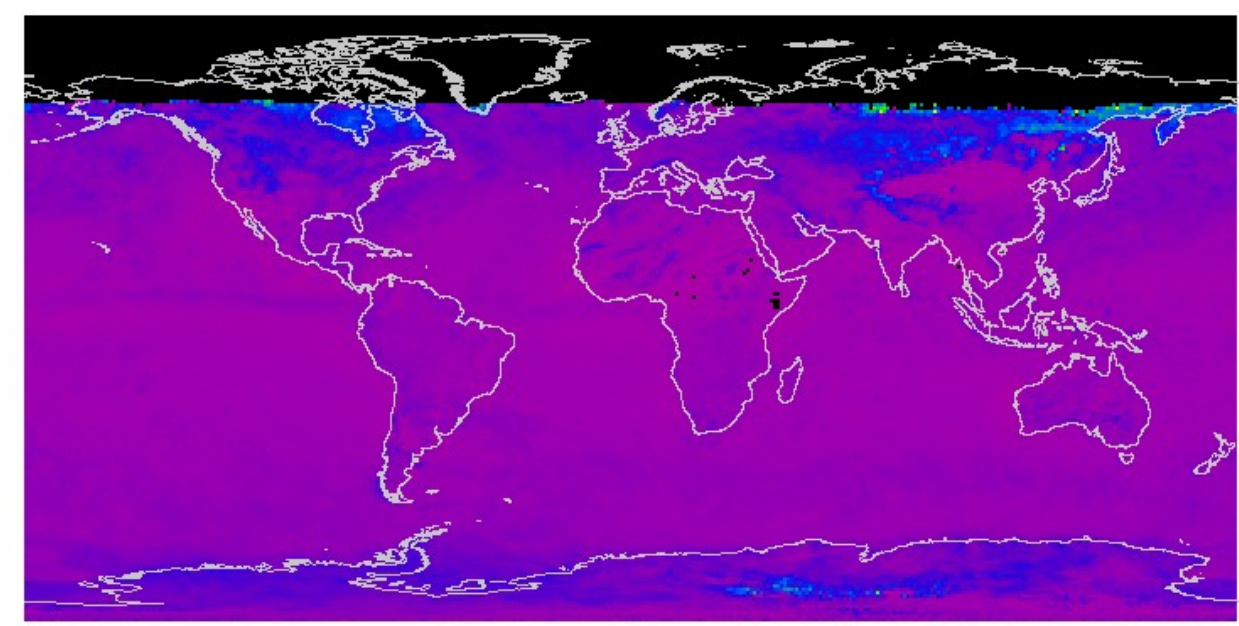

Archive Set 1372

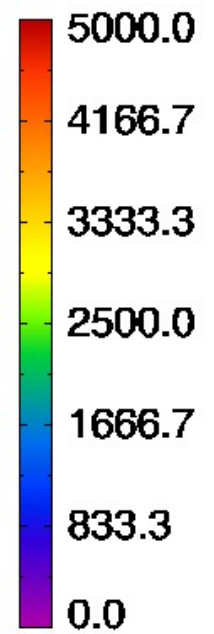

$$
\text { units = "g/m^2" }
$$




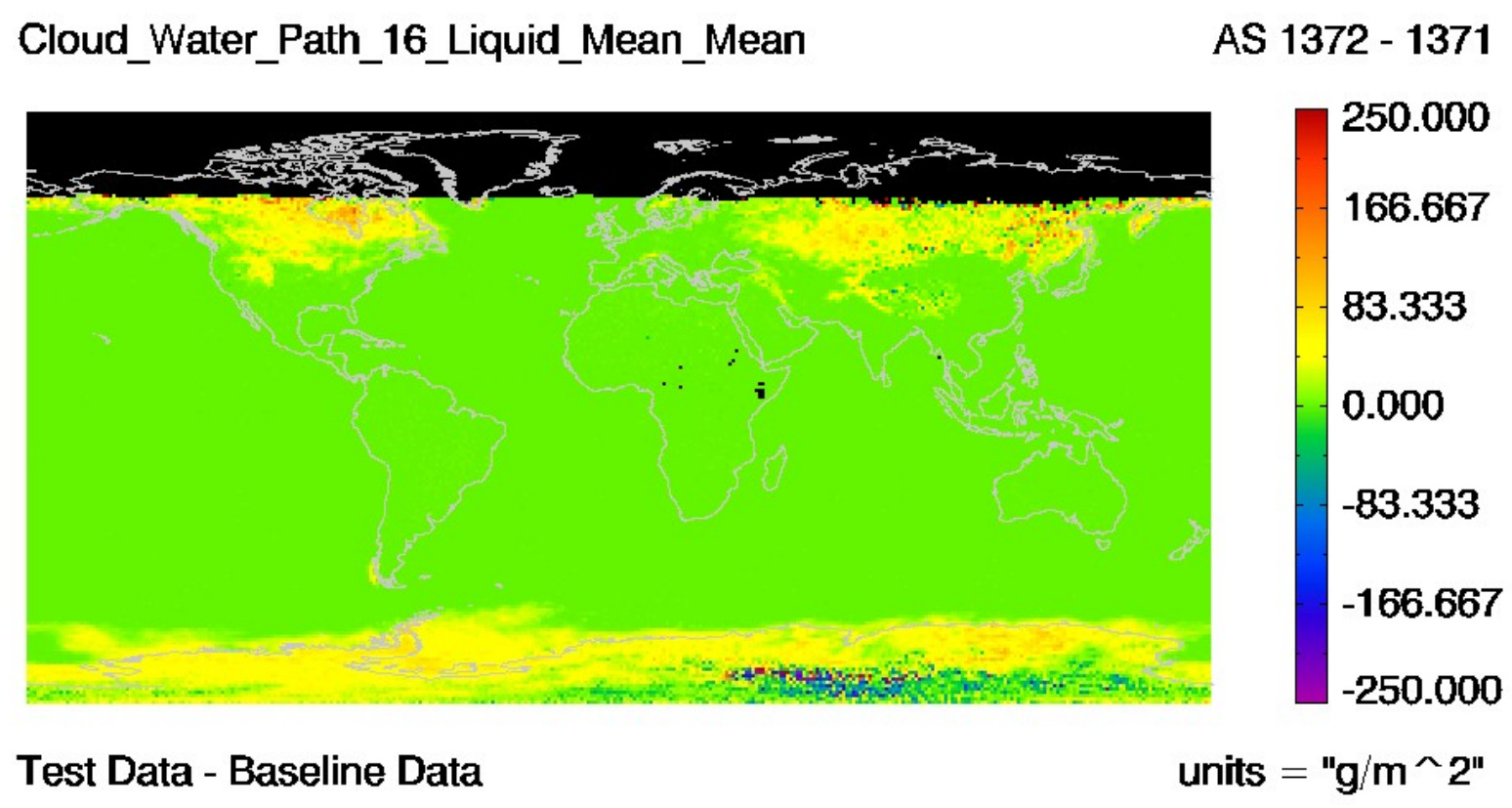

Figure 9 Cloud liquid water path (a) [B5off, B26off]] and (b) [B5off, B26off]- [B5on, B26on]. At high latitudes the difference can reach $10 \%$.

\section{DISCUSSION}

Atmospheric science products that use B5 and B26 directly will of course be affected by changes in them. But also products that use these bands as ancillary tests, e.g. for surface classification, and any product that depends on the cloud mask may also be affected.

Globally, the aerosol science products created by the aerosol-over-land Dark Target algorithm show only a very small effect as a result of the B5 correction. Locally, over ocean off of Africa the aerosol-over ocean product for the month shown, the change in optical depth $\Delta \tau$ can be up to $10 \%$ of the retrieved optical depth $\mathrm{t}(\Delta \tau / \tau=-0.01 / 1.0)$.

The TCWV change was small globally and locally, with a slight difference between the already small over-water and overland changes.

Cirrus cloud fraction can both increase and decrease as a result of B26 change, depending on underlying surface type.

Several cloud products change by $\sim 10 \%$ from a $\sim 1 \%$ change in B5 and $0.4 \%$ change in B26. This happens when pixel values are near thresholds and the correction moves reflectances across the threshold. It may also happen when auxiliary tests are used, e.g. to test for land cover type, and the land classification get changed, e.g. from water to sea ice.

\section{CONCLUSIONS}

The effect of Terra MODIS Band 5 and Band 26 corrections on downstream atmospheric science products has been evaluated for a limited set of test cases. These corrections change the B5 and B26 reflectances respectively by about $1.2 \%$ and $0.4 \%$ relative in late mission. Downstream atmospheric science products can then be affected. In particular, products using classification based on thresholds can show locally non-linear changes in product results. Preliminary investigation of science product of a small sample of months and years suggest the changes in aerosol and TCWV products are globally small, but locally can reach several percent due to thresholds and changing number of pixels included in retrievals. The monthly average of some cloud products can change locally by tens of percent from the approximately $1 \%$ relative reflectance change. 
Acknowledgement. We thank Bill Ridgway for help with the MODAPS processing.

\section{REFERENCES}

[1] Xiong, X., Sun, J., Barnes, B., Salomonson, V., Esposito, J., Erives, H., and Guenther, B., "Multiyear On-Orbit Calibration and Performance of Terra MODIS Reflective Solar Bands", IEEE Trans. Geosci. Remote Sens., vol. 45, issue 4, pp. 879-889, 2007.

[2] Xiong, X., King,, M.D., Salomonson, V., Barnes, B., Wenny, B. Angal, A., Wu, A., Madhavan, S. and Link, D., "Moderate Resolution Imaging Spectroradiometer on Terra and Aqua Missions", John Wiley \& Sons, Ltd, vol. 9781118945179, pp. 53-89, 2015.

[3] Sun, J., Xiong, X., Angal, A., Chen, H., Wu, A., and Geng, X., Time-Dependent Response Versus Scan Angle for MODIS Reflective Solar Bands, IEEE Trans. Geosci. Remote Sens., VOL. 52, NO. 6, 2014.

[4] Angal, A., A. Wu, X. Xiong, X. Geng, D. Link, and H. Chen, "On-orbit performance of the MODIS SWIR bands", Proc. SPIE 9264, Earth Observing Missions and Sensors: Development, Implementation, and Characterization III, 92641Q, (2014).

[5] Angal, A., Xiong, X., Sun, J., and Geng, X.,, On-orbit noise characterization of MODIS reflective solar bands, J. Appl. Remote Sens. 0001; 9(1):094092, 2015.

[6] Doelling, D., Hong, G.,Morstad, D., Bhatt, R., Gopalan, A., Xiong, X., The characterization of deep convective cloud albedo as a calibration target using MODIS reflectances, Conference Volume 7862, Earth Observing Missions and Sensors: Development, Implementation, and Characterization, (2010).

[7] MODIS Level-3 MODIS Atmosphere Monthly Global Product http://modis-atmos.gsfc.nasa.gov/MOD08_M3/

[8] MODIS Tropospheric Aerosol ATBD http://modis-atmos.gsfc.nasa.gov/_docs/ATBD_MOD04_C005_rev2.pdf

[9] Levy, R.C., Mattoo S., Munchak, L. A., Remer, L.A. Sayer, A. M., Patadia, F., and Hsu, N.C. The Collection 6 MODIS aerosol products over land and ocean, Atmos. Meas. Tech., 6, 2989-3034, 2013.

[10] Deep Blue aerosol project webpage http://deepblue.gsfc.nasa.gov/

[11] MODIS TCWV ATBD. http://eospso.gsfc.nasa.gov/sites/default/files/atbd/atbd_mod03.pdf

[12] MODIS MOD35 Cloud Mask ATBD http://modis-atmos.gsfc.nasa.gov/_docs/atbd_mod06_old.pdf 